\title{
Catastrophe Disguised as a Complication After Epiduroscopy- A Case Study
}

\author{
David Abejón, MD, PhD \\ Eva M Monzón, MD \\ Alberto Ríos, MD \\ Cristina Abad, MD \\ Gemma Márquez, MD \\ Alejandro Zamora, MD \\ Mar Jiménez, MD \\ Javier Carrascoso, MD
}

Background: Epiduroscopy is a minimally invasive technique that enables diagnosis and treatment within the epidural space with direct vision. Previously reported complications of this technique have been mostly mild neurological complications of a transitory nature. In the present case, we describe a serious complication following an epiduroscopy, resulting in death.

Case Report: The patient was a 39-year-old woman with lower back and lower limb pain who had undergone 3 surgical interventions previously on the lumbar spine. Only limited results from conservative and interventional treatment had been observed. She underwent an epiduroscopy in the Pain Management Unit. Immediately after the procedure, which was completed without incident, the patient presented neurological symptoms with areflexic paraplegia and a loss of sensation in the lower limbs and the upper left limb. Imaging tests identified signs of craniospinal hypotension that progressed slowly despite medical intervention. Ventricular dilation and cerebral edema without remission occurred, which ultimately caused the patient's death 2 months after admission to the intensive care unit.

Conclusion: Epiduroscopy has the potential to present adverse effects and complications. A thorough evaluation of the clinical history and the imaging tests are advisable

Key words: Complications, epiduroscopy, low back pain, spinal cord, spinal endoscopy

\section{BACKGROUND}

According to the 2006 World Initiative on Spinal Endoscopy (WISE) (1), epiduroscopy is defined as a new minimally invasive technique that allows for visualization of the epidural space with a flexible endoscope introduced through the sacral hiatus. The technique makes it possible to visualize normal anatomical structures and pathological structures, enabling direct treatment within the epidural space. Although the first epiduroscopy was described in 1931, the technique significantly advanced in the 1990s with the adoption of a flexible endoscope that enables better visualiza-

\footnotetext{
From: Hospital Universitario Quirónsalud San Jose, Madrid, Spain

Corresponding Author: Eva M. Monzón, MD, E-mail: draevamonzon@gmail.com

Disclaimer: There was no external funding in the preparation of this manuscript.

Conflict of interest: David Abejon is a consultant for Boston Scientific, PRIM, Cardiva, Abbott, and Medtronic. Eva M Monzón is a speaker for Cardiva. Alberto Ríos, Cristina Abad, Gemma Márquez, Alejandro Zamora, Mar Jimenez, and Javier Carrascoso have no conflicts of interests.

We received approval from the Ethics Committee "Comité de ética de la investigación de la Fundación Jiménez Diaz": EO010-20_HUQM.
} 
tion of the epidural space, considerably reducing complications arising from the technique (2). This gives epiduroscopy advantages over other treatments used in lumbar pathology. For example, the origin of the pain can be examined, epidural adhesions can be cleaned or released, and medication can be deposited under direct visualization with greater precision (3).

As with any invasive technique, there are still complications that can occur. Most of these are minor and transient, with a dural puncture being the most frequent. However, in other cases, major complications have been described. These include infection, pain in the puncture area, transient subdural block, and a cerebrospinal fluid (CSF) leak (4). The most serious complications that have been described are retinal hemorrhages, due to the infused volume and the speed of administration, as well as rhabdomyolysis from injecting the contrast medium $(5,6)$.

We present the first case in which a series of complications resulted in the patient's death 2 months after being admitted to critical care after an epiduroscopy in a patient with postlaminectomy syndrome. We received approval from the Ethics Committee to present this case.

\section{CASE}

The patient in the present case was a 39-year-old woman who underwent an epiduroscopy to treat postlaminectomy syndrome. The patient had previously undergone back surgery with final results of L4-S1 lumbar arthrodesis. She required reintervention twice due to dislocation of the material and had a CSF leak during the last intervention performed.

Following various interventional techniques and adjustment of the pharmacological treatment being used, it was decided to perform a lumbar epiduroscopy due to the poor results of the previous techniques and the lack of response to medical treatment.

Conforming with the usual protocols and after the informed consent form had been signed, a peripheral venous route was channelled, and, 30 minutes before the procedure, $2 \mathrm{~g}$ of intravenous cefazolin was administered as an antibiotic prophylaxis. The procedure was performed under conscious sedation. The technique was performed with strict aseptic measures. With the patient in the prone position, prior preparation of the field, and cleaning with chlorhexidine, a caudal approach was carried out with a fluoroscopic aid. Access to the caudal epidural space was performed using an 18-gauge Tuohy needle and confirmed with 2 radiological projections, anteroposteriorly (AP) and laterally, with no contrast medium used. After confirmation that the epidural position was correct, the needle was removed, keeping the guide in the canal. Following this, the Seldinger technique was used until the flexible epiduroscope was positioned in the epidural space.

During the epiduroscopy, radiculitis and fibrosis were observed in the epidural space, and the left S1 root was released with a Fogarty balloon after checking for greater involvement of the root and concordance of the patient's pain with said metamere. The procedure was concluded by administering $80 \mathrm{mg}$ of triamcinolone and $2 \mathrm{~mL}$ of $0.25 \%$ bupivacaine to reduce possible inflammation of the root treated via the caudal approach. In total, $180 \mathrm{~mL}$ of saline was administered, and the technique was performed without incident.

At the end of the procedure, a neurological examination was performed, in which the patient displayed clinical areflexic paraplegia and a loss of sensation in the lower limbs and upper left limb. Emergency magnetic resonance imaging (MRI) was performed, which showed no changes from the previous one except for the drugs along the $\mathrm{S} 1$ root.

The next day, the patient was unable to cough or phonate with airway involvement, and orotracheal intubation was performed. A new MRI was performed, showing a pattern of CSF leak, with the traction of the roots and low cranial nerves, meningeal hyperintensity, and venous engorgement (Fig. 1). An inadvertent puncture of the dura was suspected, so a blood patch was performed at the L2-L3 level. There was some clinical improvement, with some mobility in the upper left limb and phonatory capacity being regained. There was no change in terms of the distal paraplegia.

On the third day after the procedure, the patient's clinical condition worsened again. This time the MRI showed greater cervical and lumbar venous engorgement, more fluid in the epidural space, mainly at the lumbar level, as well as tetraventricular hydrocephalus and cervical ependymal canal dilation (Fig. 2). These new findings suggested a CSF disorder. However, no fistula was identified. Therefore external ventricular drainage was placed at 12 $\mathrm{mmHg}$, and a new blood patch was performed, this time at the caudal level in an effort to position the volume in the area of the possible puncture.

This time, the patient displayed little clinical improvement, even though a subsequent MRI showed radiological improvement of the hydrocephalus. One week after the procedure, due to poor progress, the imaging tests 



Fig. 1. Cervicodorsal MRI A.Sagittal. T2 Sequence. Prior to the procedure. B. Sagittal. T2 Sequence. MRI after the procedure. Epidural venous plexus engorgement. White arrows. C. Sagittal. T1 Sequence with intravenous contrast medium. MRI after the procedure. Suggestive of changes of CSF leak with an increase in dural enhancement. White arrows.

Abbreviations: CSF, cerebrospinal fluid; MRI, magnetic resonance imaging

were reviewed and a cervical pseudomeningocele was located.

An isotopic cisternography was performed in which the radiotracer was observed to stop at the level of the fourth ventricle. It did not progress to the spinal cord nor convexity with an early appearance of renal silhouettes that could suggest a CSF leak, as previously suspected.

Definitive peritoneal ventricular drainage was placed without any intraoperative incidents. However, the procedure was complicated by a right cervical hemifacial edema in the postoperative period, with a deterioration of the patient's neurological clinical status and hydrocephalus in addition to cerebral edema. The electromyography (EMG) showed established damage to the general preganglionic motor neurons of the lower limbs and upper left limb, with active denervation due to an acute axonal injury.
An attempt was made to stabilize the patient, and a myelloradiography was performed. This was discontinued after the absence of CSF in the dural sac was confirmed.

The low-pressure peritoneal ventricle drainage was replaced, and the external rescue drain was changed. The low-pressure peritoneal ventricular drainage optimally functioned with an incline of $45^{\circ}$. Therefore, an attempt was made to raise the headboard several times. The patient's clinical status worsened due to interlocking that occurred from the lack of CSF in the dural sac. Due to this and the confirmation of transtentorial herniation on the imaging results, a posterior fossa craniectomy was performed. This failed to improve her clinical condition. Her clinical condition continued to worsen and hydrocephalus progressed. Additionally, despite the external drainage, cerebral edema increased and became widespread. 


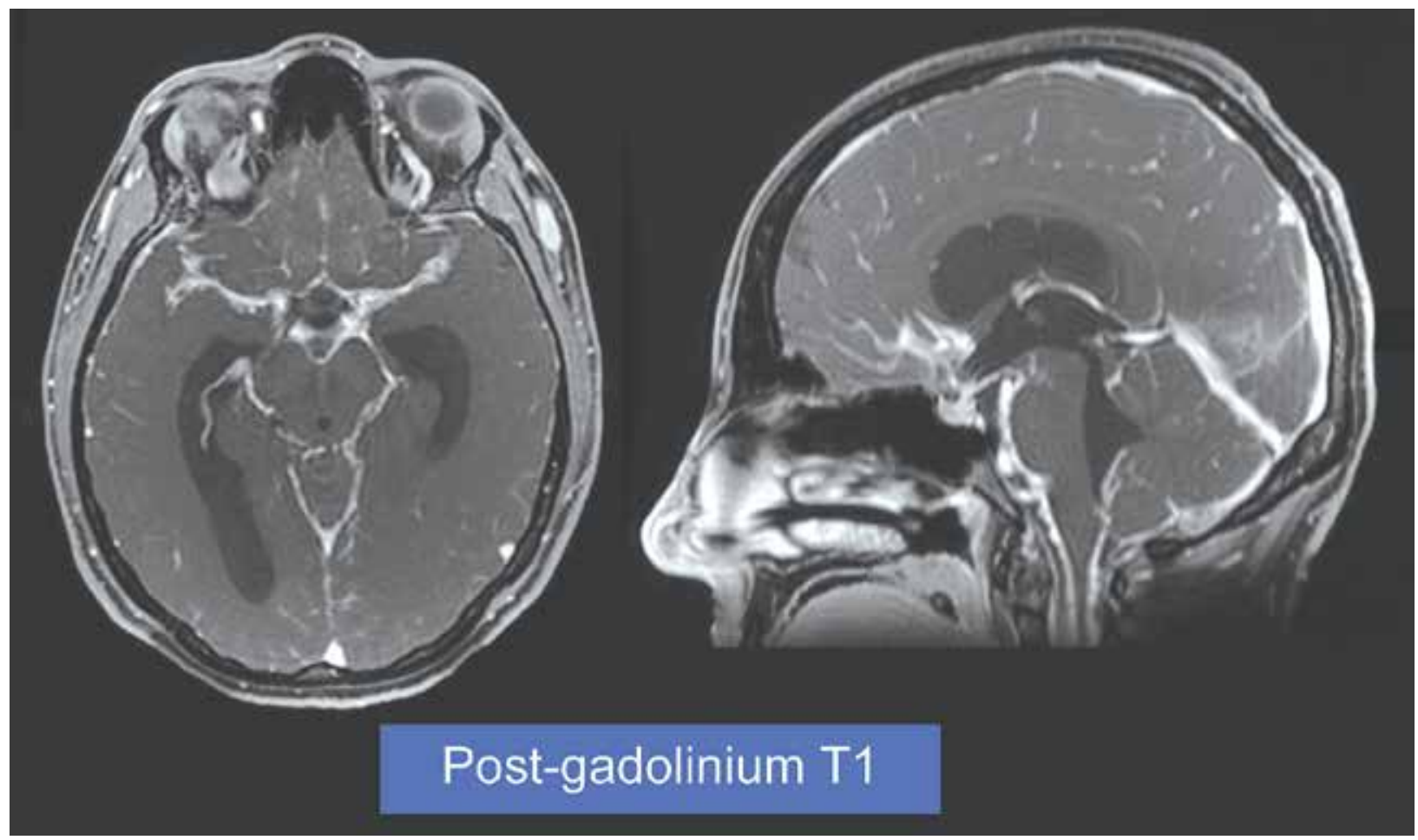

Fig. 2. Cranial MRI T1 sequence with intravenous contrast medium, axial and sagittal. Tetraventricular hydrocephalus with marked dural enhancement, suggesting loss of CSF and cerebral hypotension.

Abbreviations: CSF, cerebrospinal fluid; MRI, magnetic resonance imaging

The therapeutic actions were then limited, and the patient died 2 months after admission to the intensive care unit.

\section{DISCUSSION}

The complications that have been described in the literature after an epiduroscopy are generally minor and transitory. A systematic review conducted on 391 patients found only 14 cases of serious complications (7), such as retinal hemorrhages, or encephalopathy and rhabdomyolysis due to the contrast medium injection (5).

A dural puncture is the most frequent complication, with its incidence being higher in patients who have undergone multiple interventions. It can occur due to the puncture needle, as in any epidural technique, or with the epiduroscope or other catheters used when trying to release adhesions. This type of complication is not considered serious. The postpuncture headache rate is lower than in other populations, perhaps due to the existence of scars, adhesions, and the infusion of fluid during the procedure that can help decrease the loss of CSF (8). Treatment does not vary regardless of the procedure in which the dural puncture takes place (4).

In contrast, patients with epidural fibrosis present with an increase in intrinsic vascularization, inflammation, and vascular stasis that can sometimes explain the pain that occurs in the distension of the epidural space (9).

In the present case, in the immediate postoperative period, while the patient was in resuscitation, an acute ischemic lesion was ruled out, and no dural puncture data was visible. The first lumbar MRI only showed fluid or gas secondary to the procedure.

The worsening of the patient's clinical condition progressed and she did not respond to the treatment administered. The case was multidisciplinarily and interdisciplinarily discussed to develop some kind of aetiopathogenic hypothesis so that an appropriate treatment plan could be devised. Two main working hypotheses were developed. The first and most likely was a CSF circulation disorder, possibly due to a prior, altered condition in which a presumed fistula and/or an increase in epidural volume after epiduroscopy occurred, with secondary development 
of hydrocephalus due to a local reabsorption disorder, hyperproduction, and possible reduction of circulation to the spinal space. The second hypothesis was the venous congestion produced in the posterior epidural space that prevented the correct drainage of the CSF from the third ventricle to the subarachnoid space, with consequent retrograde hydrocephalus.

An exhaustive analysis of the serial imaging taken showed the existence of a small meningocele at the cervical level that had not been previously reported. It could have been a consequence of surgical manipulation and loss of CSF from any of the patient's previous interventions.

This case has allowed us to learn and introduce changes in our usual practice. Our patient selection has been refined, with the technique not being performed on patients who have had a loss of CSF in previous interventions. Another lesson from this case is that the entire spine should be studied, even if the patient does not report dorsal or cervical pathology.

Finally, we wish to emphasize that, although these complications have not been previously published, the necessary precautions before undertaking this interventional technique are necessary.

\section{CONCLUSION}

Epiduroscopy, similar to all interventional techniques, has the potential to result in adverse effects and complications. To prevent serious complications such as the one described in this article, we recommend a thorough evaluation of the background and imaging tests of the spine. Additionally, maximum safety conditions for performing this type of procedure must be maintained.

\section{REFERENCES}

1. Beltrutti D, Groen GJ, Saberski L, Sandner-Kiesling A, Schütze G, Weber G. Epiduroscopy: Consensus Decision March, 2006. The Pain Clin [Internet] 2007; 19:47-50.

2. Saberski LR, Kitahata LM. Direct visualization of the lumbosacral through the sacral hiatus. Anesth Analg 1995; 80:839-840.

3. Kallewaard JW, Vanelderen P, Richardson J, Van Zundert J, Heavner J, Groen GJ. Epiduroscopy for patients with lumbosacral radicular pain. Pain Pract 2014; 14:365-377.

4. Manchikanti $L$. The value and safety of epidural endoscopic adhesiolysis. Am J Anesth 2000; 27:275-279.

5. Mizuno J, Gauss T, Suzuki M, Hayashida M, Arita H, Hanaoka K. Encephalopathy and rhabdomyolysis induced by iotrolan during epiduroscopy. Can J Anesth 2007; 54:49-53.

6. Gill J, Heavner J. Visual impairment following epidural fluid injections and epiduroscopy: A review. Pain Med [Internet] 2005; 6:367-374.

7. Avellanal M, Diaz-Reganon G, Orts A, Gonzalez-Montero L, De Andrés Ares J. Epiduroscopy: Complications and troubleshooting. Tech Reg Anesth Pain Manag 2014; 18:35-39.

8. Avellanal M, Diaz-Reganon G. Interlaminar approach for epiduroscopy in patients with failed back surgery syndrome. Br J Anaesth 2008; 101:244-249.

9. Raffaeli W, Righetti D, Andruccioli J, Sarti D. Epidural space and chronic pain. Eur J Pain Suppl 2010; 4:269-272. 
